well-referenced methodological sections dealing with the logistics of experimentation in the male reproductive sciences.

To all proud possessors of a $\mathrm{Y}$ chromosome the final chapter on the influence of environmental factors on spermatogenesis and sperm function should be of special interest. The chapter covers a wide range of interactions, including those between addictive drugs, therapeutic agents, food additives, industrial chemicals and pesticides. The list of compounds interfering with male reproductive function is indeed alarmingly long and may go some way towards explaining the apparent progressive decline in the sperm densities encountered in normal fertile men from a mean of $100 \times 10^{6}$ cells per millilitre twenty years ago to a value of $50 \times 10^{6}$ at the present time. The role of such environmental hazards in the aetiology of male infertility is obviously in urgent need of assessment.

The emphasis of the book is clearly a reflection of the interests of the authors. Thaddeus Mann's contribution to our understanding of the biochemistry and function of semen has been universally acknowledged and his work has inspired a generation of scientists, including myself. One consequence of this interest in semen, however, is that the behaviour of spermatozoa once they have escaped from the seminal plasma and initiated their quest for the female gamete(s) is not given the same depth of treatment. The abilities of spermatozoa to migrate through the female reproductive tract and engage in the process of fertilization are of critical importance to the definition of fertility, yet neither of these important properties can be accurately predicted from the behaviour in sperm in seminal plasma.

A related problem is that the population of spermatozoa participating in fertilization in vivo spend a majority of their lifespan in a state of so-called capacitation. In contrast, a significant proportion of the studies reviewed in the extensive chapter on sperm biochemistry have been carried out on spermatozoa of uncertain physiological status suspended in simple, artificial media. The authors are obviously aware of this problem but conclude that it in no way detracts from the value and biological significance of the results. I wish I could share their confidence.

The layout of the book is excellent, although certain passages might have benefited from more extensive illustration, and I wish there had been a concluding chapter in which the authors' knowledge and experience could have been used to highlight the most significant recent developments. Nonetheless this is a major work by two highly distinguished authors; it should find a home on the bookshelves of anyone professing an interest in male reproductive physiology.

\title{
Favourite recipes for the macrophage chef
}

\section{S. Gordon}

Manual of Macrophage Methodology:

Collection, Characterization, and Function. Edited by H.B. Herscowitz et al. Pp.514. ISBN 0-8274-1222-6. (Dekker: 1981.) SwFr. 185, $\$ 65$.

METCHNIKOFF drew attention to the specialized phagocytic capability of macrophages a century ago. Apart from their role in immunity against many important intracellular pathogenic organisms, the macrophages distributed throughout the body secrete numerous products responsible for inflammation, repair and injury. In addition, these cells retain differentiated traits in vitro and thus provide ideal material to study growth, membrane traffic, destruction of tumour cells and many other topics of interest to both cell biologists and immunologists.

Description of methods for studying the macrophage has traditionally been relegated to immunology texts, but several attempts are now under way to produce a separate, specific manual. This volume has arrived early and does contain much useful information, but is badly flawed by lack of a proper sense of where macrophage research is heading. Although sound prescriptions are given for the isolation of macrophages and study of some of their activities, exciting areas like $\mathrm{H}_{2} \mathrm{O}_{2}$ production and surface antigens are overlooked. Individual authors often turn to outmoded methods of cell characterization and elimination, by silica for example, rather than coming to grips with the problem of adherent cell heterogeneity. Thus, the implications of the discovery of the potent "antigen presenting" activity of dendritic cells, a minor contaminant of most macrophage populations, are not confronted. Other points of emphasis are irregular; interleukin-1 is well covered, but not CSF; the recovery of macrophages from spleen by enzyme digestion, a difficult but important procedure, is neglected; pinocytosis mediated by specific receptors is ignored; and so on.

Some authors give workmanlike assessments of procedures otherwise scattered in research papers. These include a catalogue of permanent cell lines with macrophage markers, culture methods for bone marrow precursors, cytochemical procedures for the light microscope, and the ever-useful detailing of Fc and complement receptor assays. Nevertheless, there are too many descriptions of everyone's

In celebration of its fiftieth anniversary the American Institute of Physics has published a handbook for physicists of all persuasions, the Physics Vade Mecum. The book consists of 22 chapters containing useful formulae, numerical data, definitions and references pertinent to various fields, and is available from AIP Marketing Services, 335 East 45 Street, New York, price $\$ 25$. favourite assay for microbicidal/cytocidal activity of activated macrophages and not enough assessment of their value. Readers will therefore find this book only moderately useful, and not sufficiently critical, integrated or comprehensive.

S. Gordon is Reader in Experimental Pathology at the Sir William Dunn School of Pathology, University of Oxford.

\section{Chemical dynamics}

\section{J.N. Murrell}

Potential Energy Surfaces and Dynamics Calculations for Chemical Reactions and Molecular Energy Transfer. Edited by Donald G. Truhlar. Pp.866. ISBN 0-306-40755-8. (Plenum: 1981.)\$85, £53.55.

IN A rapidly developing area of science it is usually necessary to go to the primary literature to obtain details about the currently active experimental or theoretical techniques. The reason for this is not just that textbooks take some time to be published, but that authors have difficulty in deciding the opportune moment to write them. This book consists of a collection of original papers that were given at a symposium in August 1980. In that respect it provides an up-to-date perspective on this field and, in my view, a broadly balanced one.

Researchers interested in potential surfaces and molecular dynamics are concerned with understanding and predicting the results of collisions between simple molecules. The equations necessary for these predictions are well established but it is only in recent years that computational techniques have reached the level of providing, in many cases, reliable predictions. There are two stages in the calculation, both of which have seen recent advances: first, it is necessary to have accurate potential functions, and second they must be used for accurate dynamics.

There are three papers in the volume which give an overview of particular aspects of the field. These are on unimolecular dynamics, reactive scattering and non-reactive scattering. In addition there are 33 papers which deal largely with particular molecular systems. The contributions are generally of good quality and by respected authors, and thus I can thoroughly recommend the book for purchase by libraries and by active research workers in the field.

J.N. Murrell is a Professor in the School of Chemistry and Molecular Sciences, University of Sussex. 